\title{
Prehospital deaths in the Yorkshire Health Region
}

\author{
D Limb, A McGowan, J E Fairfield, T J D Pigott
}

\begin{abstract}
Objectives-To estimate the proportion of prehospital deaths in a British population of trauma victims which may be preventable, and to investigate the effect of death at the scene and death in transit on potential survivorship.

Methods-Blinded review, by four specialists with an interest in trauma, of necropsy results and details of age, sex, and mechanism of injury for prehospital trauma deaths in the Yorkshire Health Region in a 12 month period.

Results-Complete records were traced on 305 of 337 trauma deaths, 190 being recorded as dead on arrival of emergency services and 115 dead on arrival at hospital. In the group declared dead at the scene, three of the four assessors considered $93 \%$ of deaths to have been inevitable and only $2 \%$ as potential survivors $(25 \%$ of this group sustaining inevitably fatal injuries such as brain avulsion or decapitation). In the group dead on arrival $81 \%$ were felt to be inevitable deaths and $5 \%$ potential survivors.

Conclusions-There seems to be less scope for salvage of victims of trauma death in a British population than has been recorded in America, possibly due to a higher proportion of blunt trauma deaths here. Those who die in transit consist of a less severely injured group with a higher potential for survival.

( $\mathcal{F}$ Accid Emerg Med 1996;13:248-250)
\end{abstract}

Key terms: prehospital death; major trauma; fatal accidents; necropsy

Level 5,

Clinical Sciences

Building,

St James's University

Hospital.

Beckett Street,

Leeds LS9 7TF:

Department of

Orthopaedics

D Limb

Accident and

Emergency

Emergency

Department

Department of

Anaesthetics

J E Fairfield

Correspondence to:

Mr D Limb,

Senior Registrar in

Orthopaedics.
While it is likely that the level of care provided in trauma centres can improve the outcome following major trauma, considerable debate still surrounds the prehospital care of these patients. Knowledge of the caseload and spectrum of injuries is essential for planning of services, in the development of the role of paramedic teams, and public training in basic resuscitation skills. The ultimate aim is to reduce the preventable death rate, but at present we do not know what proportion of prehospital deaths truly could have been avoided given optimally trained prehospital personnel and public awareness of airway care. We looked at all prehospital trauma deaths in the Yorkshire Region in one year in an attempt to identify what proportion of patients could have survived given optimal care.

\section{Methods}

Data collected for a study of the major trauma caseload of the Yorkshire Health Region between October 1988 and September 1989 were reviewed, focusing on those cases which died before arrival at hospital. Sixteen hospitals were involved and the accident and emergency (A\&E) records were reviewed retrospectively in order to identify trauma cases. Initial identification was also made from the resuscitation room register, trauma ward admissions book, mortuary and pathologists' registers, and coroners' records. Injuries caused by electrocution, drowning, poisoning, asphyxia, and hypothermia were excluded, as were cases from incidents occurring outside the boundaries of the region who were brought in for specialist or continuing care.

For each case identified, the age, sex, details of times of incident (where known), and cause of incident were recorded. The necropsy results were summarised to describe the anatomical extent of the injuries sustained. No information was available regarding the state of health of any individual before the trauma episode.

These data were reviewed independently by an orthopaedic surgeon, an accident and emergency physician, a neurosurgeon, and an anaesthetist. Each, while blinded to the opinions of the other reviewers, gave an opinion as to whether the patient could have survived their injuries, given cardiopulmonary care by general public bystanders and prehospital care by a trained paramedic team. The deaths were categorised into "potential survivor", "definite death", and "unclear/insufficient information".

The four sets of independent opinions were then read in conjunction to ascertain the degree of agreement between specialists on the most likely outcome in each case. For each patient the number of "potential survivor" and "definite death" responses was recorded.

\section{Results}

There were 337 prehospital deaths due to trauma in the Yorkshire Region between October 1988 and September 1989. Complete records were traced on 305 of these $(91 \%)$. Of these, 190 were recorded as being found "dead at scene" (DAS), and 115 "dead on arrival" (DOA).

In the DAS group there were 144 males and 46 females. The median age was 39 years (range 2 to 87 years). In the DOA group there were 84 males and 31 females. The median age was 40 years (range 6 to 91 years). The mechanism of injury is given by category in table 1 . 
Table 1 Mechanism of injury

\begin{tabular}{lll}
\hline Mechanism & $\begin{array}{l}\text { Dead at scene } \\
\text { (number) }\end{array}$ & $\begin{array}{l}\text { Dead on arrival } \\
\text { (number) }\end{array}$ \\
\hline Road traffic accident & 91 & 96 \\
Self inflicted injury & 46 & 7 \\
Assault & 22 & 2 \\
Home accident & 18 & 5 \\
Work accident & 12 & 3 \\
Sport & 1 & 2 \\
\hline
\end{tabular}

The results of independent review by the four assessors are presented for the two groups of patients in tables 2 and 3. Overall $113(63 \%)$ were judged to have suffered inevitably fatal injuries and only three $(2 \%)$ were judged unanimously to be potential survivors in the DAS group. In the DOA group the figures were $60(50 \%)$ and one $(<1 \%)$ respectively. However, in some cases not all the assessors felt able to give an opinion. In cases where three or more opinions were put forward [177 $(93 \%)$ in the DAS group and $109(95 \%)$ in the DOA group], the number of cases felt to have suffered inevitably fatal injuries or to be potential survivors by at least three assessors was calculated: $157(89 \%)$ in the DAS group and $109(81 \%)$ in the DOA group were felt to be definite deaths, while only four $(2 \%)$ in the DAS group and five $(5 \%)$ in the DOA group were felt to be potential survivors.

The level of agreement between assessors was addressed. Firstly, in the DAS group all four assessors gave the same opinion in 113 cases considered to be definite deaths and in three cases of potential survivors. In a further 21 cases, more than one assessor felt unable to give an opinion but the opinions of the remaining assessors were in total agreement, bringing the level of full agreement to 134 cases (71\%). Secondly, in the DOA group, 60 were considered to have suffered inevitably fatal injuries and one was judged to be a potential

\section{Table 2 Assessments of cases found dead at scene}

Levels of total agreement in assessment of outcome in 190 cases found dead at the scene

$\begin{array}{lc}4 / 4 \text { agree on likely outcome } & 120(72 \%) \\ 3 \text { agree } & 17(9 \%) \\ 2 \text { agree } & 3(2 \%) \\ 1 \text { opinion } & 1(0.5 \%) \\ \text { Total giving an opinion in agreement } & 141(74 \%)\end{array}$

with that of all other assessors

Disagreements on likely outcome in cases found dead at scene

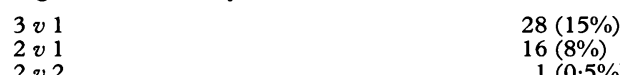

Three out of four assessors were therefore in agreement that a case was either a "definite death" or a "definite survivor" in $137+28=165$ cases $(87 \%)$

Table 3 Assessments of cases found dead on arrival at hospital

Levels of total agreement on outcome in 115 cases who died in transit to hospital

\begin{tabular}{|c|c|}
\hline $\begin{array}{l}4 / 4 \text { agree on outcome } \\
3 \text { agree } \\
2 \text { agree } \\
1 \text { opinion }\end{array}$ & $\begin{array}{c}58(50 \%) \\
9(8 \%) \\
7(6 \%) \\
7(6 \%)\end{array}$ \\
\hline \multicolumn{2}{|c|}{$\begin{array}{l}\text { Disagreements on likely outcome in cases who died in transit } \\
\text { o hospital }\end{array}$} \\
\hline $\begin{array}{lll}3 & v & 1 \\
2 & v & 1 \\
1 & v & 1 \\
2 & v & 2\end{array}$ & $\begin{array}{c}24(21 \%) \\
8(7 \%) \\
6(5 \%) \\
6(5 \%)\end{array}$ \\
\hline
\end{tabular}

survivor by all four. There were 23 further cases where all assessors giving opinions were in total agreement, bringing the total to 84 cases $(70 \%)$.

The greatest variability in opinion arose when a head injury was present, with a more optimistic opinion given by the neurosurgeon in these cases. This is particularly notable where three of the assessors felt a case to have suffered inevitably fatal injuries but one considered the case a potential survivor. This accounted for 28 cases in the DAS group and 21 cases in the DOA group. The neurosurgical opinion was the single (more optimistic) opinion in 27 of the 28 disagreements in the DAS group and 19 of the 21 the disagreements in the DOA group.

Some patients had single injuries which in isolation were judged to be incompatible with survival, and the distribution of this type of injury was different in each group. Decapitation, avulsion of the brain, avulsion of the heart, and burns exceeding $80 \%$ of the body surface in a person over 70 years of age were considered unsurvivable. Forty nine patients $(25 \%)$ in the DAS group and nine $(8 \%)$ in the DOA group fell into this category.

\section{Discussion}

The proportion of prehospital deaths following major trauma that could be avoided given optimal public awareness of basic resuscitation techniques and advanced paramedic care is not known. It has been suggested that up to 39\% of these deaths could be avoided by basic airway care alone, ${ }^{1}$ and other studies have provided similarly optimistic figures for the effects of advanced life support techniques administered by paramedic staff. ${ }^{23}$ While it is attractive to predict that this is the level of reduction of prehospital deaths that could be brought about by increasing public awareness of basic resuscitation techniques, in reality even with optimal public awareness and paramedic availability, we feel that the impact on prehospital death rate could not be this great. Our figures do not support this contention, with a much lower rate of potential survival being noted than in previous studies. This is particularly so for the group classified as "dead at scene" (that is, on arrival of the emergency services). Twenty five per cent of these casualties were found to have at least one totally unsurvivable insult, together with a spectrum of associated injuries which would render any form of resuscitation pointless. In view of this invariably high mortality in the DAS group we believe that studies should point out the proportion of patients considered who were diagnosed as being dead at the scene of the accident and those who showed vital signs at the scene of injury, but were dead on arrival at the emergency care facility. Those "dead at scene" consist of a much more severely injured population with a lower potential salvage rate.

We would also emphasise the requirement for a neurosurgical assessment in studies such as this, with the greatest discrepancy of opinion arising between the neurosurgeon and other 
specialists. It is accepted, however, that in any study such as this there is considerable interrater variability. ${ }^{4}$ The inconsistency in this study arose not simply because of opposing opinions, but was due to the nature of the dataset. Necropsy records detail the presence of haematoma and cerebral injury, but only describe the volume and location of such injuries in general terms. The nonneurosurgical assessors were more ready to assign a case to either the "potential survivor" or "definite death" group rather than classify as "insufficient information", resulting in levels of agreement of only three out of four assessors. In almost all of these cases the neurosurgeon gave the most optimistic opinion as it was felt that the haematoma or cerebral injury could not be taken into account without more information on its extent.

This study considers only deaths from major trauma and the potential impact of improved prehospital care on these. It is suggested that the reduction in mortality may not be so great as has been hoped, as has previously been suggested. ${ }^{5}$ However, we have excluded deaths from drowning, electrocution, poisoning, asphyxia, and hypothermia and this may be the field where these services could have the greatest effect in reducing unnecessary mortality.

1 Hussain LM, Redmond AD. Are prehospital deaths from accidental injury preventable? BMF 1994;308:1077-80.

2 Aprahamian C, Thompson BM, Towne JB, Darin JC. The effect of a paramedic system on mortality of major open intra-abdominal vascular trauma. $\mathcal{f}$ Trauma 1983;23: 687-90.

3 Baxt WG, Moody P. The impact of advanced prehospital emergency care on the mortality of severely brain-injured patients. F Trauma 1987;27:365-9.

4 MacKenzie EJ, Stein Wachs DM, Bone LR, Fiocare DJ, Ramzy AI. Inter-rater reliability of preventable death judgements. $\mathcal{F}$ Trauma 1992;33:292-302.

5 Sampalis JS, Lavoie A, Williams JI, Mulder DS, Kalina M. Impact of on-site care, prehospital time and level of inhospital care on survival in severely injured patients. $f$ Trauma 1993;34:252-61.

6 Anderson IWR, Black RJ, Ledingham IMcA, Little K, Robertson CE, Urquhart JD. Early emergency care study: the potential and benefits of advanced prehospital care. BMF 1987:294:228-31.

\section{The Faculty of Accident and Emergency Medicine}

\section{SPECIALTY EXAMINATION}

The first Faculty specialty examination in accident and emergency medicine will be held on 3/4 October 1996 at the Royal College of Physicians and Surgeons of Glasgow.

Regulations and application forms are available from:

The Secretariat

Intercollegiate Specialty Boards

3 Hill Square

Edinburgh

EH8 (DR

Tel: 01316629222

Fax: 01316629444

The closing date for receipt of applications is 9 August 1996

Fee $-£ 500$ 